\title{
Research on Trajectory Planning of 6-DOF Cutting-robot in Machining Complex Surface
}

\author{
Sheng-xi Jiao ${ }^{1}$, Hao Wang ${ }^{1}$, Lin-lin Xia ${ }^{2}$ and Shuai Zhang ${ }^{1}$ \\ ${ }^{1}$ University of Northeast Electric Power in Jilin, Advanced Sensor Technology Institute, Jilin, China \\ ${ }^{2}$ University of Northeast Electric Power in Jilin, Research Institute of robot and image visual detection technology, Jilin, China
}

\begin{abstract}
It is important and difficult for the complicated surface processing in mechanical industry. In this paper, an improved algorithm for trajectory planning is proposed in impeller surface processing by using 6-DOF cutting-robot. Taking a single finished path of the impeller blade as an example, the feedrate of the cutter, bow height error, cutterorientation and position are planned by the B-spline interpolation algorithm, the best cutting trajectory is obtained. On the basis of trajectory planning, the optimal movement scheme of 6-DOF cutting-robot joints is obtained, the 6-DOF cutting-robot feedrate and trajectory smooth transition is achieved and the joints movement adaptive adjustment is completed. Finally, the angles, the angular velocitys of the joints and their interrelated properties are analyzed. The research works indicate that the robot joint angle curves are continuous and stable, which has met the requirements of smooth movement of the robot, and the results show that the trajectory planning is effective and practical.
\end{abstract}

\section{Introduction}

The impeller is one of the most important components of the widely used centrifugal compressor[1]. At present, cemented carbide impeller is processed by five axis machining centers mostly. It is suitable for machining high precision workpiece and its cost is expensive[2]. Multi degrees of freedom industrial robot has its unique advantages in machining complex surface workpieces, which has low hardness and low machining precision. Compared with five axis machining center, six degree of freedom cutting robot cutter control is more flexible, and complex surface trajectory control is easier, so we can machine workpieces with cutting robot according to accuracy requirements at lower processing costs $[3,4]$. This kind of application requires the robot has high accuracy position and orientation, reasonable path planning and robot joints movement optimal control[5, 6].Therefore, it is very necessary to research cutting tools trajectory planning and robot joints movement control[7, 8].

Valente A (2017) proposes a new approach for defining the motion profiles of robots, based on a smooth trajectory generation model [9]. Avram O, and Valente A (2016) studied the trajectory planning for reconfigurable industrial robots designed to operate in a high precision manufacturing industry [10]. Xu Z (2014) plans the trajectory with Bezier Curve in Cartesian space for industrial gluing robot[11]. Shi B H and He J P (2016) present a new velocity control algorithm and three BSpline curve Instantaneous velocity interpolation algorithm for open Welding robot system based on
LinuxCNC [12]. Gou Z J and Wang C (2014) apply fifthorder B-spline interpolates to plan robot trajectory when interpolation points and the kinematic parameters are known, and the trajectory is simulated and validated by the software of ADAMS [13]. Lin Y (2017) investigates a robot posture optimization methodology based on robotic performance indexes [14]. Li S (2014) presents a method of robotic machining trajectory automatic generation for complex surface grinding and polishing, the NonUniform Rational B-splines (NURBS) curve fitting and adaptive sampling algorithm are taken as core of the method [15].

In the above studies, the trajectory planning algorithms are complicated and not easy to implement, mostly are universal algorithms, there is no algorithm for trajectory planning and interpolation of robot machining impellers. In this paper, a strategy of trajectory planning in robot operation space is introduced, the industrial robot end-effector's positions and orientations are planned, and the finishing of the impeller's blade is realized. In order to achieve a balanced transition of trajectories, B-spline interpolation algorithm is used. The algorithm makes advantage of curvature and bow height error to achieve the feedrate adaptive adjustment.

\section{Design of six-dof cutting robot model}

In this paper, ABB IRB-140 industrial robot is selected as the simulation model, as shown in fig. 1. The end-effector of robot three-dimensional model is consisted by a small diameter ball milling cutter and a high speed spindle, the rest of the main components of the model are the same as 
real robot in space layout. The IRB-140 industrial robot is a series robot with six joints, each joint includes shaft, bearing, sleeve, gear, motor, and reducer. In order to ensure that the simulation model meets the requirements of motion simulation and reduces the amount of simulation calculation, some parts of the robot model need to be simplified, joints are simplified into axes, other parts are remain unchanged which include the base coordinate system and the earth coordinate system, the high speed spindle and the ball milling cutter. The high speed spindle and the ball milling cutter are fixed connection, by controlling the drives on the six joints, it is possible to get the best cutter- position and orientation of the robot.

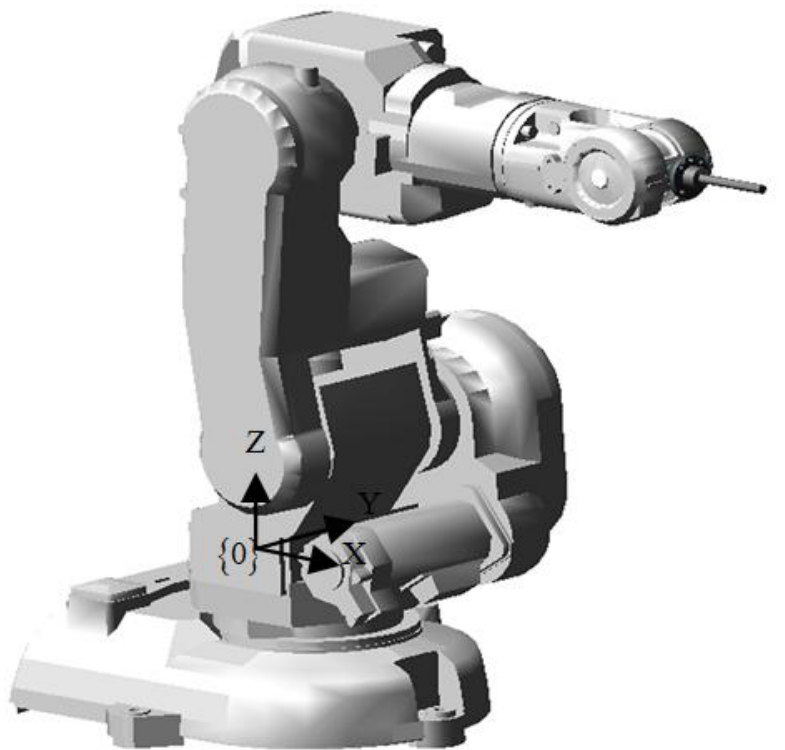

Figure 1. Three-dimensional model of cutting industrial robot.

\section{Kinematics analysis of robot}

IRB-140 industrial robot has six degrees of freedom, we define $\{\boldsymbol{n}, \boldsymbol{o}, \boldsymbol{a}, \boldsymbol{m}\}$ as the cutter- position and orientation of the robot. The cutter-position refers to the center of the cutter tip, which is simply called the cutter center position. m denotes the cutter center position, ${ }^{0} \boldsymbol{m}=\left[\begin{array}{lll}m_{x} & m_{y} & m_{z}\end{array}\right]^{\mathrm{T}}$. The transformation matrix of the coordinate system $\{T\}$ of the cutter with respect to the base coordinate system $\{0\}$ is [16]:

$$
{ }_{6}^{0} T={ }_{1}^{0} T_{2}^{1} T_{3}^{2} T_{4}^{3} T_{5}^{4} T_{6}^{5} T
$$

Its position and orientation transformation matrix ${ }_{6}^{0} T$ is:

$$
{ }_{6}^{0} T=\left[\begin{array}{cccc}
n_{x} & o_{x} & a_{x} & m_{x} \\
n_{y} & o_{y} & a_{y} & m_{y} \\
n_{z} & o_{z} & a_{z} & m_{z} \\
0 & 0 & 0 & 1
\end{array}\right]
$$

The position and orientation transformation matrix denotes the relationship between the cutter- position and orientation and the angle of joints, and the Jacobian matrix of the robot can be established by the matrix ${ }_{6}^{0} T$. The Jacobian matrix denotes the velocity mapping between the Cartesian space and the joint space. In the base coordinate system $\{0\}$, we define $\boldsymbol{m}_{\boldsymbol{e}}$ as the feedrate vector of cutter, the feedrate vector of cutter can be expressed by the following formula:

$$
\boldsymbol{m}_{e}=J_{m}(q) \dot{q}
$$

In which, the parameter $q$ is the velocity of joints, and the matrix $J_{m}(q)$ is the Jacobian matrix of cutter in the base coordinate system. According to the reference, the Jacobian matrix of 6 -DOF industrial robots is a $3 \times 6$ partial derivative matrix:

$$
J_{m}(q)=\left[\begin{array}{llllll}
\frac{\partial m_{x}}{\partial q_{1}} & \frac{\partial m_{x}}{\partial q_{2}} & \frac{\partial m_{x}}{\partial q_{3}} & \frac{\partial m_{x}}{\partial q_{4}} & \frac{\partial m_{x}}{\partial q_{5}} & \frac{\partial m_{x}}{\partial q_{6}} \\
\frac{\partial m_{y}}{\partial q_{1}} & \frac{\partial m_{y}}{\partial q_{2}} & \frac{\partial m_{y}}{\partial q_{3}} & \frac{\partial m_{y}}{\partial q_{4}} & \frac{\partial m_{y}}{\partial q_{5}} & \frac{\partial m_{y}}{\partial q_{6}} \\
\frac{\partial m_{z}}{\partial q_{1}} & \frac{\partial m_{z}}{\partial q_{2}} & \frac{\partial m_{z}}{\partial q_{3}} & \frac{\partial m_{z}}{\partial q_{4}} & \frac{\partial m_{z}}{\partial q_{5}} & \frac{\partial m_{z}}{\partial q_{6}}
\end{array}\right]
$$

\section{Finishing path and cutter-orientation calculation}

The blade tool path of finishing operations are displayed in fig. 2, the impeller blade is processed by ball end milling according to planned trajectories, the dot milling processing method is as fig. 3 shows. The ball end milling method is a method in which the surface of blades is processed by the ball cutter according to the flow direction of the blades, this method of processing has the characteristics of processing non-ruled surface leaves, and the processing operations are not limited by the type of blade.

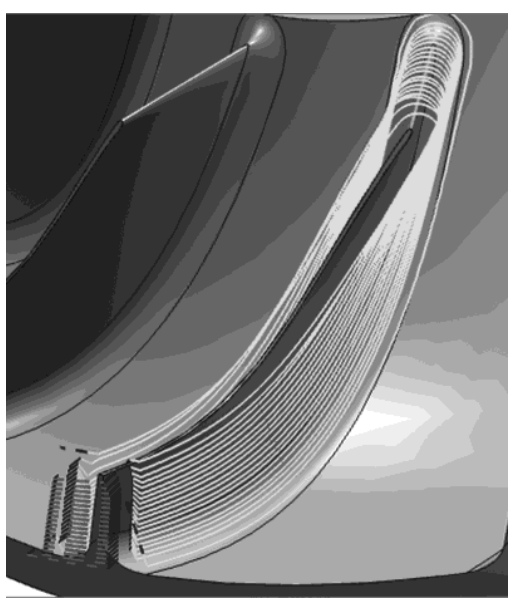

Figure 2. The blade tool path of finishing operations.

${ }^{0} \boldsymbol{m}$ and ${ }^{0} A$ are extracted from the post-processing file of UG. In the base coordinate system, the position vector of the cutter is defined as 
${ }^{0} \boldsymbol{m}=\left[\begin{array}{lll}m_{x} & m_{\mathrm{y}} & m_{\mathrm{z}}\end{array}\right]^{\mathrm{T}}$, and the orientation vector of the cutter is defined as ${ }^{0} A=\left[\begin{array}{lll}i & j & k\end{array}\right]^{\mathrm{T}}$. The orientation vector of the cutter is also called the cutter axis vector. In order to describe the cutter- position and orientation of the simulation model quickly and easily, it is necessary to describe the cutter- position and orientation using the XYZ sequence Euler angle [17]. First of all, the rotation matrix formula of XYZ sequence Euler angle is shown in equation (5).

$$
\begin{aligned}
& \boldsymbol{R}_{x y z}\left(q_{x}, q_{y}, q_{z}\right)=\boldsymbol{R}_{x}\left(q_{x}\right) \boldsymbol{R}_{y}\left(q_{y}\right) \boldsymbol{R}_{z}\left(q_{z}\right) \\
& =\left[\begin{array}{ccc}
1 & 0 & 0 \\
0 & \cos q_{x} & -\sin q_{x} \\
0 & \sin q_{x} & \cos q_{x}
\end{array}\right]\left[\begin{array}{ccc}
\cos q_{y} & 0 & \sin q_{y} \\
0 & 1 & 0 \\
-\sin q_{y} & 0 & \cos q_{y}
\end{array}\right]\left[\begin{array}{ccc}
\cos q_{z} & -\sin q_{z} & 0 \\
\sin q_{z} & \cos q_{z} & 0 \\
0 & 0 & 1
\end{array}\right] \\
& =\left[\begin{array}{cccc}
\quad \cos q_{y} \cos q_{z} & -\cos q_{y} \sin q_{z} & \sin q_{y} \\
\cos q_{x} \sin q_{z}+\sin q_{x} \sin q_{y} \cos q_{z} & \cos q_{x} \cos q_{z}+\sin q_{x} \sin q_{y} \sin q_{z} & -\sin q_{x} \cos q_{y} \\
\sin q_{x} \sin q_{z}-\cos q_{x} \sin q_{y} \cos q_{z} & \sin q_{x} \cos q_{z}+\cos q_{x} \sin q_{y} \sin q_{z} & \cos q_{x} \cos q_{y}
\end{array}\right]
\end{aligned}
$$

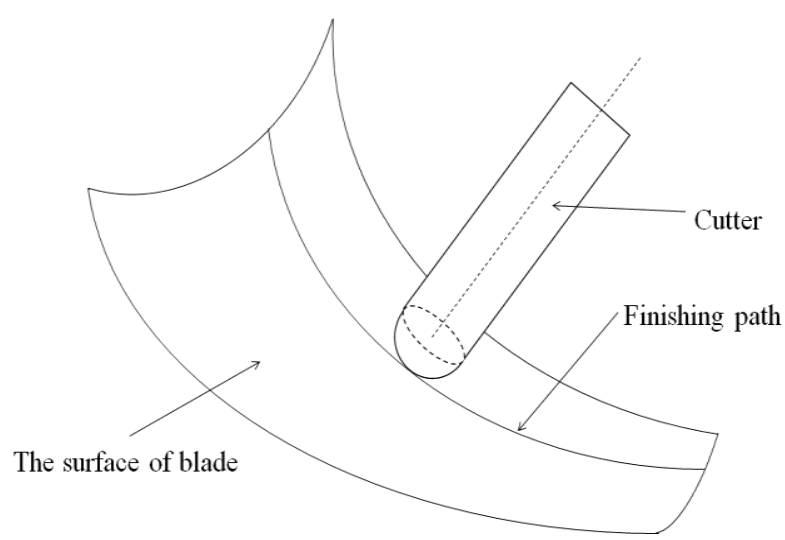

Figure 3. The point milling process.

Secondly, the Euler angle can be calculated by the following formula:

$$
{ }_{6}^{0} T=\boldsymbol{R}_{x y z}\left(q_{x}, q_{y}, q_{z}\right)
$$

Finally, the Euler angle can be got:

$$
\left\{\begin{array}{l}
q_{x}=l \tan \left(-2 a_{y}, 2 a_{z}\right) \\
q_{y}=l \sin \left(a_{x}\right) \\
q_{z}=l \tan \left(-2 a_{x}, 2 n_{x}\right)
\end{array}\right.
$$

\section{Feedrate planning}

\subsection{Planning algorithm}

Planning the feedrate of the cutter is for smoothing the cutting and ensuring the robot run smoothly. An improved cubic non-uniform B-spline curve interpolation algorithm is put forwarded, the improved algorithm is used to plan the trajectory of Cartesian coordinates space, a single path is taken as an example to carry out trajectory planning.

Supposing that the number of tool center point and tool axis vectors for a single path is $n$, $\boldsymbol{m}_{i}\left(x_{i}, y_{i}, z_{i}\right)$ and $\boldsymbol{m}_{i}^{\prime}\left(i_{i}, j_{i}, k_{i}\right)(i=0, \mathrm{~K}, n-1)$ respectively, the parameter $\boldsymbol{m}_{i}^{\prime}$ is the unit vector. In order to carry out cutter axis vector optimization easily [18], the cutter axis vector can be transformed by the following formula

$$
\left\{\begin{array}{l}
w_{i}=x_{i}+i_{i} \\
u_{i}=y_{i}+j_{i} \\
v_{i}=z_{i}+k_{i}
\end{array}\right.
$$

Defining $m_{i}^{\prime}\left(w_{i}, u_{i}, v_{i}\right)$ as the point of the cutter axis, which means the end point of the cutter axis.

The equation of the cubic non-uniform B-spline curve can be expressed by the following formula formula[19] :

$$
C(u)=\sum_{i=0}^{n} N_{i, p}(u) D_{i}
$$

In the formula (9), $u$ is the curve parameters, $D_{i}$ is the control vertex vector, $N_{i, p}(u)$ is the B - spline curve basis function, the $p_{i}(i=0, \mathrm{~K}, n-1)$ is defined as the value data, the cutter center position points and the cutter axis points can be planned respectively by using the same node vector.

In the B-spline curve interpolation algorithm, the centripetal parameter method is used to construct $U\left(u_{0}, \mathrm{~L}, u_{n+5}\right)$ which defined as the node vector, the value of the first node is $u_{0}=u_{1}=u_{2}=u_{3}=0$, and the value of the end node is $u_{n+2}=u_{n+3}=u_{n+4}=u_{n+5}=1$. In addition, the value of the intermediate node can be calculated by the following formula: 


$$
u_{3+i}=u_{2+i}+\frac{\sqrt{\left|p_{i+1}-p_{i}\right|}}{\left(\sum_{i=0}^{n-2} \sqrt{\left|p_{i+1}-p_{i}\right|}\right)}
$$

Control vertices are calculated by type points $U\left(u_{0}, \mathrm{~L}, u_{n+5}\right)$, they are the key to anti-solution interpolation point, the value points can be calculated to control the vertices. The control vertex is defined as $d_{i}(i=0, \mathrm{~K}, n+1)$, which can be calculated by equation (10):

$$
p_{i}=C\left(u_{3+i}\right)=\sum_{j=i}^{3+i} N_{j, 3}\left(u_{3+i}\right) d_{j}
$$

By further derivation of equation (11), the $p_{i}$ is converted as:

$$
p_{i}=\left[\begin{array}{c}
N_{i, 3}\left(u_{3+i}\right) \\
N_{1+i, 3}\left(u_{3+i}\right) \\
N_{2+i, 3}\left(u_{3+i}\right) \\
N_{3+i, 3}\left(u_{3+i}\right)
\end{array}\right]^{\mathrm{T}}\left[\begin{array}{llll}
d_{i} & d_{i+1} & d_{i+2} & d_{i+3}
\end{array}\right]^{\mathrm{T}}
$$

There are eight control vertices, but only six control vertices can be calculated by equation (12). In order to calculate the other two control vertices, two boundary condition formulas need to be added:

$$
\left\{\begin{array}{l}
d_{0}=p_{0} \\
d_{n+1}=p_{n-1}
\end{array}\right.
$$

$d_{i}$ and $d_{i}^{\prime}$ can be computed by equation (12) and (13), $d_{i}$ is defined as the control vertex of the cutter center position B-spline curve, and $d_{i}^{\prime}$ is defined as the control vertex of cutter axis B-spline curve.

In order to make the robot run smoothly, and meet the complicated and varied characteristics of the blade processing path, the acceleration-uniform speeddeceleration interpolation program is used and the cubic B-spline algorithm is improved [20]. In the improvement of B-spline interpolation algorithm, the curvature and bow height error are introduced to adaptive adjusting the federate. The formula (14) and formula (15) of the improved algorithm are improved.

In formula (14), the curvature of the path and the realtime feed rate are taken into the formula, and the bow height error ER is calculated. In formula (15), the actual feed speed is divided into two segments. First, the curvature of the path and the error of the height of the bow are brought into the formula. Then the calculated value is compared with the maximum feed speed $\mathrm{F}$, and the suitable feed speed is selected.

$$
\begin{gathered}
E R=\rho_{i}-\sqrt{\rho_{i}^{2}-\left(\frac{v(t) T}{2}\right)^{2}} \\
V\left(u_{i}\right)=\left\{\begin{array}{l}
F, \frac{2}{T} \sqrt{\rho_{i}^{2}-\left(\rho_{i}-E R\right)^{2}}>F \\
\frac{2}{T} \sqrt{\rho_{i}^{2}-\left(\rho_{i}-E R\right)^{2}}, \frac{2}{T} \sqrt{\rho_{i}^{2}-\left(\rho_{i}-E R\right)^{2}} \leq F
\end{array}\right.
\end{gathered}
$$

\subsection{Interpolation results analysis}

In this paper, the single path contains 141 position and orientation data value points. The values of the feedrate, acceleration and interpolation cycle are $\mathrm{F}=25 \mathrm{~mm} / \mathrm{s}$, $\mathrm{a}=250 \mathrm{~mm} / \mathrm{s} 2$ and $\mathrm{T}=10 \mathrm{~ms}$ respectively. The programmed cutter feedrate is shown in fig. 4, the cutter feedrate is roughly divided into three sections: acceleration stage, intermediate stage and deceleration stage. From the cutter feedrate curve we can see that the maximum value of the feedrate is $25 \mathrm{~mm} / \mathrm{s}$. In the deceleration stage, the feedrate slows down near to $7 \mathrm{~mm} / \mathrm{s}$, and then accelerate to the maximum value. The curve shows that the feedrate of the cutter has adjusted adaptively according to the curvature of the path.

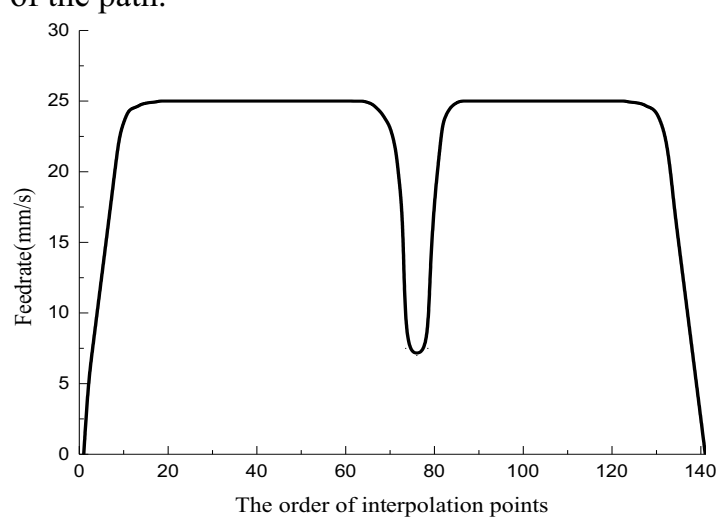

Figure 4. feedrate curve.

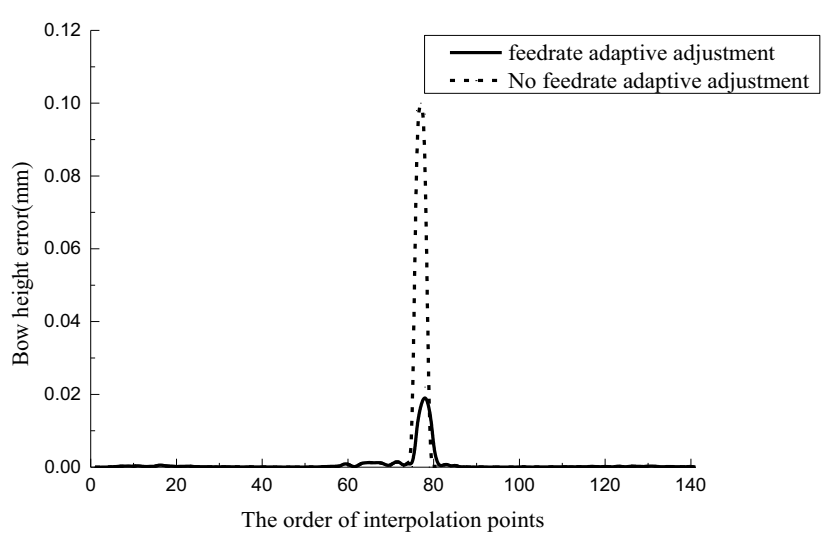

Figure 5. bow height error curve.

Bow height error changes as shown in fig. 5, the dotted line shows that the value of the bow height error can reach $0.1 \mathrm{~mm}$, while the solid line shows that the maximum value of the bow height error is $0.02 \mathrm{~mm}$. The error data has proved that after the feedrate adaptive adjustment algorithm [21] is added, the bow height error 
is obviously reduced and the machining accuracy is improved. Fig. 4 and fig. 5 prove that the improved algorithm can make the cutting steady, the feedrate of the mutation and the impact is reduced.

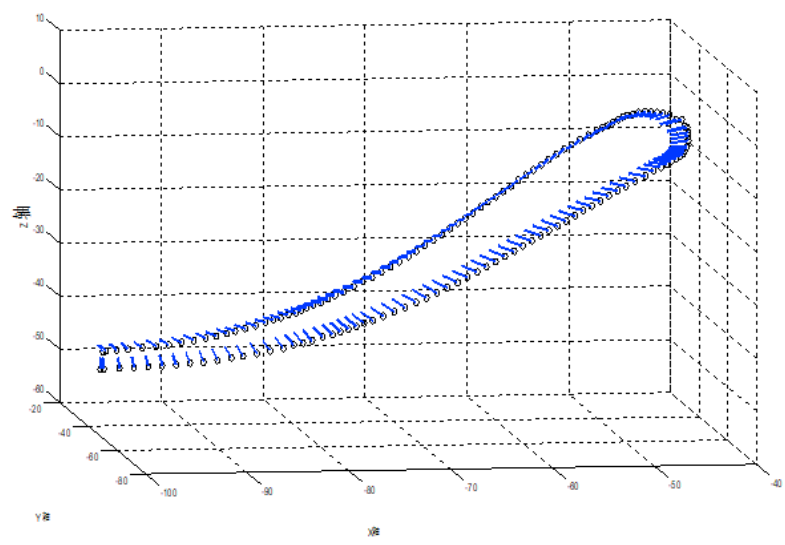

Figure 6. Three-dimensional graph of cutter-orientation and position.

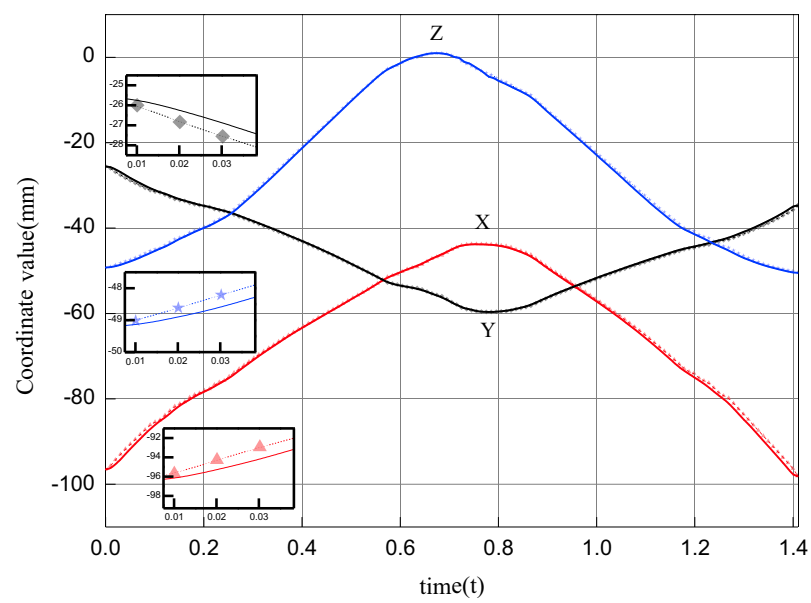

Figure 7. The graph of cutter center position.

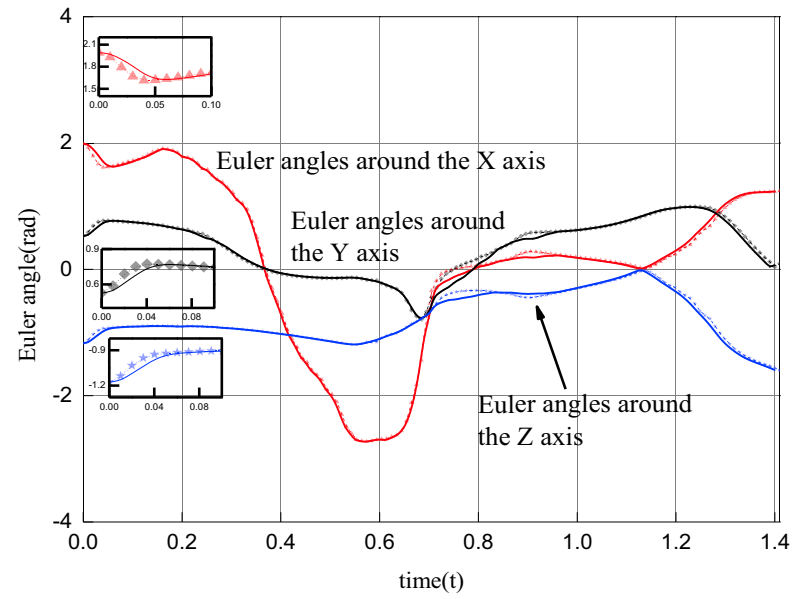

Figure 8. The graph of cutter-orientation.

After using the improved B-spline interpolation algorithm, the cutter center position points and the cutter axis points are planned, and the three-dimensional trajectory of cutter after trajectory planning is obtained, as fig. 6 shows. Fig. 6 proves that the movement process of robot cutter is continuous and smooth after trajectory planning. Fig. 7 shows the cutter center position curve and fig. 8 shows the cutter-orientation curve. From fig. 7 and fig. 8, comparing the data before and after the trajectory planning, it can be seen that the curve becomes smooth, in which the dotted line indicates the trajectory before planning and the solid line indicates after the trajectory planning. From the partial enlargement in fig. 7 and fig. 8 , it can be seen that the cutter center position curve and the cutter-orientation curve after trajectory planning has obvious acceleration and deceleration effect, this also proves the conclusion shown in fig. 6 , and the acceleration and deceleration process is consistent with the process shown in fig. 4 .

\section{Kinematics simulation and analysis}

The simulation time and simulation steps are to be set as $1.41 \mathrm{~s}$ and 200 steps respectively. The six joint angle curves and joint angular velocity curves of the robot are obtained by simulation [22]. Fig. 9 shows the contrast diagram of the joint angle curves before and after the trajectory planning. In the picture, the black dotted line represents the joint angle curve before the planning, and the red solid line represents the joint angle curve after the planning. It can be seen from fig. 9, the joint angle curves after the planning are smooth and continuous, which improves the fluctuation of the robot in the initial and end positions, and the robot achieves a smooth transition in the middle transition section.

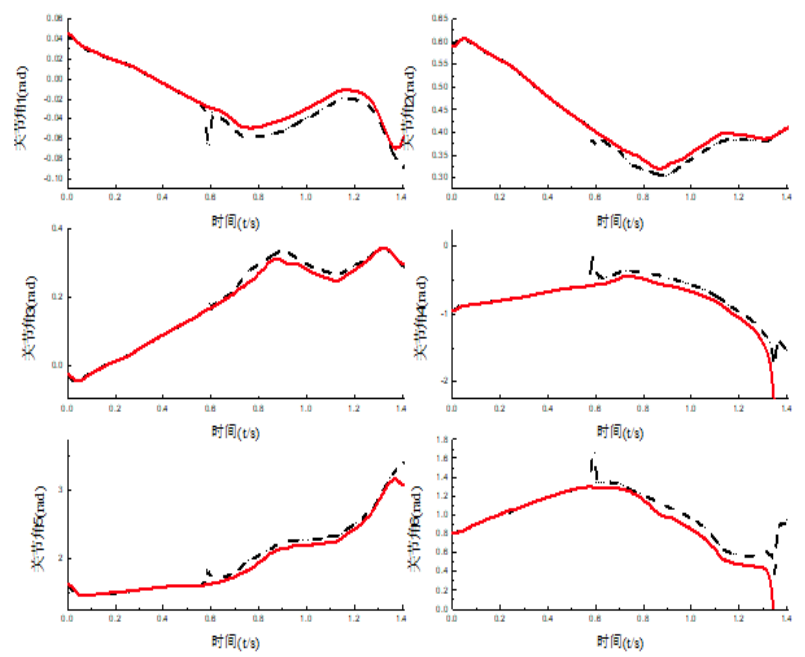

Figure 9. Comparison of the joint angle curves of the robot before and after trajectory planning.

Fig. 10 is a contrast diagram of the angular velocity curves of the robot joints before and after the planning. In the picture, the black dotted line represents the angular velocity curves before the planning, and the red solid line represents the planned angular velocity curves. The fig. 10 shows that the first and last segment of the joint curve is zero, and there is no velocity mutation after the trajectory planning. This shows that the robot can start and stop smoothly. In the middle section of the curve, the speed of the joint angle is still stable, which shows the smooth and smooth transition of the robot in the path transition section. The above 
results prove that after the trajectory planning, the cutting robot moves smoothly, which is in line with the actual operation of the industrial robot.

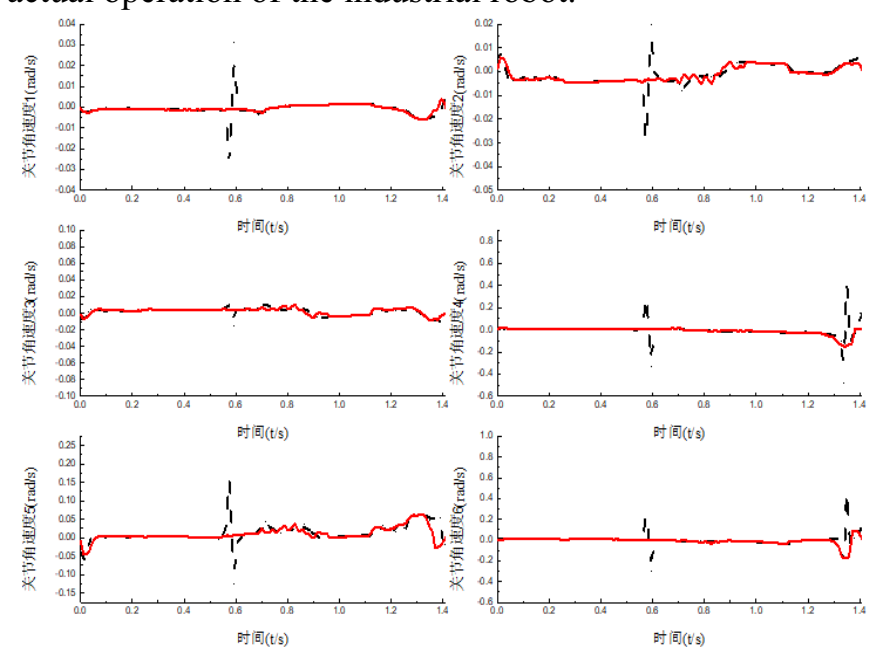

Figure 10. Comparison of angular velocity curves of robot joints before and after trajectory planning.

\section{Conclusion}

In this paper, a 6-DOF cutting-robot simulation model is built with ABB IRB-140 industrial robot as the reference prototype. Through the study of B-spline interpolation algorithm, the feedrate of cutter with the single path of the finishing of impeller's blade is planned, which makes the cutting robot run smoothly during the machining process. Through the analysis of feedrate and bow height error, it can be seen that the 6-DOF cutting-robot achieves higher accuracy. The simulation results show that the trajectory planning algorithm proposed in this paper is reasonable and efficient, the method is effective in the machining of the impeller surface and provides a reference for the 6-DOF cutting-robot to process the complex surface.

\section{Acknowledge}

The research work is supported by "National Natural Science Foundation of China: Research on information fusion strategy of vehicle MIMUs/GPS navigation system under Computational Intelligence Aided mode (61503073)"

\section{References}

1. Dong L.; Cao L. Effects of residual riblets of impeller's hub surface on aerodynamic performance of centrifugal compressors. 9:1, 99-113, DOI: 10.1080/19942060.2015.1004 813, (2015)

2. Zhang Z.; Liu Z.; Cheng Q.; et al. An approach of comprehensive error modeling and accuracy allocation for the improvement of reliability and optimization of cost of a multi-axis NC machine tool. $89: 1-4, \quad 1-19$, DOI: 10.1007/s00170-016-8981-x, (2016)
3. Olabi A.; Béarée R.; Gibaru O.; et al. Feedrate planning for machining with industrial six-axis robots. $18: 5, \quad 471-482$, DOI:10.1016/j.conengprac.2010. 01.004, (2010)

4. Iglesias I.; Sebastián M A.; Ares J E. Overview of the State of Robotic Machining: Current Situation and Future Potentia. 132, 911-917, DOI: 10.1016/j.proeng.2015.12.577,( 2015)

5. Kubela T.; Pochyly A.; Singule V. Assessment of industrial robots accuracy in relation to accuracy improvement in machining processes. 720-725, DOI: 10.1109/EPEPEMC.2016. 7752083,( 2016)

6. Kelaiaia R. Improving the pose accuracy of the Delta robot in machining operations, 91:5-8, 2205-2215, DOI 10.1007/s00170-016-9955-8,( 2017)

7. Giberti H.; Sbaglia L.; Urgo M. A path planning algorithm for industrial processes under velocity constraints with an application to additive manufacturing. $43, \quad 160-167, \quad$ DOI: 10.1016/j.jmsy.2017.03.003,(2017)

8. Bharathi A.; Dong J. Feedrate optimization for smooth minimum-time trajectory generation with higher order constraints. 82:5-8, 1029-1040, DOI: 10.1007/s00170-015-7447-x,(2016)

9. Valente A.; Baraldo S. Carpanzano E. Smooth trajectory generation for industrial robots performing high precision assembly processes. 2017, https://doi.org/ 10.1016/j.cirp.2017.04.105.

10. Avram O.; Valente A. Trajectory Planning for Reconfigurable Industrial Robots Designed to Operate in a High Precision Manufacturing Industry. 2016, 57:461-466, DOI: 10.1016/j.procir.2016.11.080.

11. Xu Z.; Wei S.; Wang N.; et al. Trajectory Planning with Bezier Curve in Cartesian Space for Industrial Gluing Robot. 146-154,( 2014)

12. Shi B H.; He J P. The robot motion trajectory algorithm research based on B-spline and new velocity planning. DOI: 10.1109/CCDC.2016. 7532065,( 2016)

13. Gou Z J.; Wang C. The Trajectory Planning and Simulation for Industrial Robot Based on Fifth-Order B-Splines. 538, 367-370, DOI: 10.4028/www. scientific.net/AMM.538.367,(2014)

14. Lin Y.; Zhao H.; Ding H. Posture optimization methodology of $6 \mathrm{R}$ industrial robots for machining using performance evaluation indexes. 48, 59-72, DOI: 10.1016/j.rcim.2017.02.002,(2017)

15. Li S.; Xie X.; Yin L. Research on Robotic Trajectory Automatic Generation Method for Complex Surface Grinding and Polishing. 124-135,(2014)

16. Siciliano B.; Sciavicco L.; Villani L.; et al. Robotics: modeling, planning and control. (2010).

17. Corke P. Robotics, Vision and Control. Springer Berlin Heidelberg, (2011).

18. Liu Y.; Hui L I.; Wang Y. Realization of a 5-axis NURBS Interpolation with Controlled Angular Velocity. 25:1, 124-130, DOI: 10.1016/S10009361(11)60370-1, (2012)

19. Liu Y.; Jin R.; Chen M.; et al. Contour propagation using non-uniform cubic B-splines for lung tumor delineation in 4D-CT. 1-13, DOI: $10.1007 / \mathrm{s} 11548-$ 016-1457-5,(2016) 
20. Vulliez M.; Lavernhe S.; Bruneau O. Dynamic approach of the feedrate interpolation for trajectory planning process in multi-axis machining. 88:5-8, 112, DOI: 10.1007/s00170-016-8903-y, (2017)

21. Sun Y.; Zhao Y.; Bao Y.; et al. A novel adaptivefeedrate interpolation method for NURBS tool path with drive constraints. 2014, 77:1, 74-81, DOI: 10.1016/j.ijmachtools. 11.002,( 2013)

22. Bian Z.; Ye Z.; Mu W. Kinematic analysis and simulation of 6-DOF industrial robot capable of picking up die-casting products. 41-44, DOI: 10.1109/AUS.2016.7748017,(2016) 